\title{
Ergativité dans le nord-est brésilien: la famille Karirí
}

\author{
Aryon Dall'Igna Rodrigues
}

\section{La famille Kariri}

La famille linguistique Karirí est un petit groupe génétique de langues attesté dans le nord-est du Brésil entre la fin du 17ème et le commencement du 19ème siècle surtout sur le bas cours du fleuve São Francisco et vers le sud de celui-ci. Aujourd'hui, ces langues sont toutes mortes et les descendants des anciens Karirí (1500 Kirirí dans la municipalité de Ribeira do Pombal, Etat de Bahia, probablement mélangés avec d'autres groupes; 1500 Karirí-Xokó dans la municipalité de Porto Real do colégio, dans l'Etat d'Alagoas) ne parlent que le portugais.

Le premier témoignage connu est dû au missionnaire jésuite italien Mamiani et comprend un catéchisme catholique bilingue (langue indigène portugais) et une grammaire (Mamiani 1698 et 1699) publiés comme étant « de la nation Kirin » et dont Bernard de Nantes précisera plus tard qu'il s'agissait plus spécifiquement de la langue des indiens Kipeá (Bernardo de Nantes 1709: a.III). Un vocabulaire de la même langue mentionné dans les relations jésuitiques sous le titre vocabularium linguae quiririorom, n'a pas été publié et n'a pas, malheureusement, été retrouvé.

Le deuxième témoignage donc est un autre catéchisme, également bilingue, composé dans la langue des indiens dzubukuá et en portugais par le missionnaire capucin français Bernard de Nantes (1709).

Un troisième témoignage est donné un siècle plus tard grâce au naturaliste bavarois von Martius, qui a recueilli deux courts vocabulaires, l'un chez les Karirí (« Cayriri ») du lieu Pedra Branca et l'autre chez les Sabujá de Caranguejo, dans la province de Bahia (Martius 1867:215-219).

La reconnaissance d'une famille linguistique Karirí est due au grand pionnier français de la linguistique sud-américaine Lucien Adam, qui a essayé 
d'organiser toutes les données linguistiques des sources écrites dans son livre Matériaux pour servir à l'établissement d'une grammaire comparée des dialectes de la famille karirí (1897). Plusieurs hypothèses ont été avancées sur la parenté de cette famille avec d'autres groupements linguistiques sud-américains. Les plus récemment publiées sont celles de Greenberg, de Kaufman et de Rodrigues. Greenberg (1987:83) l'a incluse dans le groupe équatorial, un des onze sous-groupes de son amérindien, laissant ainsi entendre qu'elle serait génétiquement plus proche d'autres sous-groupes qui s'y trouvent inclus tels que le Maipure et le Tupi. Kaufman (1990c:48; 1994:34, 51) présente la famille Karirí dans la « région géolinguistique » Brésil Nord-est sans suggérer de parenté génétique avec d'autres groupements. J’ai proposé et je soutiens encore l'association do cette famille avec les onze autres qui forment le groupe Macro-Jê (Rodrigues 1986:48-55, 1999:164-206). Du point de vue génétique, c'est encore un groupe fortement hypothétique, mais en faveur duquel on a déjà reconnu plusieurs faits de nature phonologique et lexicale et un nombre non négligeable de ressemblances typologiques dans la grammaire, qui renforcent l'hypothèse d'une origine commune (Rodrigues 1999:198-201). Il est vrai que c'est précisément dans le domaine de la typologie grammaticale que la famille Karirí se détache de la plupart des autres membres du Macro-Jê, mais en s'associant, de ce point de vue, avec un autre membre de l'ensemble, le Guató, même si celui-ci se trouve dans l'extrême sud-ouest du domaine MacroJê, tandis que le Karirí en occupait l'extrême nord-est (Rodrigues 1999:198 et passim).

Dans la présente contribution on n'utilisera que des exemples de la langue Kipeá, celle documentée dans les livres de Mamiani.

\section{Structure des phrases verbales}

On peut distinguer deux types principaux de phrases verbales :

(a) phrases intransitives: (COMPL) $\mathrm{V}_{\mathrm{IN}} \mathrm{N}_{\mathrm{EXP}}$ (COMPL)

(b) phrases transitives : (COMPL) $\mathrm{V}_{\mathrm{TR}} \mathrm{N}_{\mathrm{EXP}} n o \mathrm{~N}_{\mathrm{AG}}$ (COMPL) ${ }^{1}$

COMPL représente des compléments adverbiaux, tant des adverbes simples que des syntagmes prépositionnels, lesquels peuvent se succéder aussi bien au commencement qu'à la fin de la phrase. Le verbe précède toujours les arguments nucléaires, le nom est suivi par l'adjectif mais précédé par ses déterminants, le possesseur suit le possédé et les autres relations de dépendance sont exprimées au moyen de prépositions².

1 La liste des abréviations est donnée en fin d'article. Ou absolutif, voir 6. conclusion (note de l'éditeur).

2 Dans les traductions mot-à-mot (entre parenthèses) les prépositions seront identifiées par le nom du cas qu'elles marquent plus typiquement : ABL(ATIF), ACC(USATIF), DAT(IF), ERG(ATIF), ... 
Le mot no, qui marque la relation d'agent, est une préposition. On pourrait dire que le syntagme prépositionnel avec no exprimant l'agent constitue un complément comme n'importe quel autre et que, par conséquent, il n'y aurait qu'un seul type principal de phrase verbale. Pourtant, il y a au moins un fait qui permet de distinguer clairement les verbes transitifs et par conséquent les phrases transitives : seuls les verbes transitifs donnent lieu à deux nominalisations relalives, l'une pour le patient et l'autre pour l'agent, tandis que pour les verbes intransitifs il n'y en a qu'une seule (voir 5. ci-dessous).

\section{Marques de personne}

Pour tous les verbes, la personne de l'expérient est marquée au moyen de préfixes qui établissent cinq distinctions : le locuteur (1), l'interlocuteur (2), une tierce personne non réfléchie (3), une tierce personne réfléchie (3REFL) et une première personne inclusive, c'est-à-dire locuteur et interlocuteur(s) ensemble (1/2). Voici des exemples :
(1) hi-te-kri bo hi-era
1-venir-PERF ABL 1-maison
"je suis venu de ma maison"
(2) e-re-kri kune do e-padzu?
2-s'irriter-PERF INT DAT 2-père
"cst-ce que tu t'es irrité contre ton père?"

(3) more si-pa kradzo hi-ña di

bientôt 3-être tué vache 1-ERG FUT

"bientôt je tuerai la vache"

(4) s-uka Péro do di-bysapri

3-aimer Pierre DAT 3REFL-battre

"Pierre aime à être battu"

(5) do pa adje i-wo

ACC être tué gibier 3-aller

"il est allé pour tuer du gibier"

(6) Bo ku-nuñe no tupã i-dzene k-umarã

Pour 1/2-garder ERg Dieu 3-contre 1/2-ennemi

"afin que Dieu nous garde contre nos ennemis" 
La marque pour 3 est effacée quand le verbe est le premier mot de la frase :

(7) pa-itu kradzo no karai être tué-DUR vache ERG homme.blanc

"l'homme blanc est en train de tuer la vache"

Les marques des autres personnes peuvent aussi être effacées, mais alors les pronoms personnels correspondants occupent la position du syntagme nominal :

(8) sõ hietsã no wo

mordre moi ERG serpent

"un serpent m'a mordu"

\section{Accord de personne}

Les marques de personne sur le verbe s'accordent seulement avec le premier syntagme nominal, lequel manifeste l'expérient (INTRANSIT.) ou le patient (TRANSit.). Outre les exemples (3) et (4), voyez (9) et (10) ci-dessous :

(9) s-uka i-ñu-roe do di-padzu

3-aimer 3-enfant-mâle DAT 3REFL-père

"le fils aime son père"

(10) more si-pa kradzo hi-ña

bientôt 3-être tué vache 1-ERG

"bientôt je tuerai la vache"

\section{Réflexivisation}

Il y a une marque réfléchie (ou coréférencielle) exclusivement pour la troisième personne (3REFL), laquelle est contrôlée par le premier syntagme nominal, comme on peut le voir dans l'exemple (4) ci-dessus et dans les exemples (11) et (12) ci-dessous :

(11) potu kune judeo-a so tupã? potu dy pro, si-di pouvoir INT juif-PL ACC Dieu? pouvoir NEG CONC, 3-donner

di-pa no tupã-ho do judeo-a neru

3refl-être tué ERG Dieu-même DAT juif-PL pourtant

"est-ce que les juifs avaient pouvoir sur Dieu? encore qu'ils n'avaient pas ce pouvoir, Dieu lui-même l'a donné aux juifs pour le tuer" 
(12)

$\begin{array}{lllll}\text { pa-kri } & \text { Paulo no ñho mo } & \text { d-era } \\ \text { être tué-PERF Paul ERG Indiens dans } & \text { 3-REPL-maison } \\ \text { "les Indiens ont tué Paul dans sa maison" }\end{array}$

Comparez (12) avec (13) ci-dessous :

(13)

$\begin{array}{llllll}\text { pa-kri } & \text { Paulo no niho mo } & \text { m-era } \\ \text { être tué-PERF } & \text { Paul ERG Indiens dans } & \text { 3-maison } \\ \text { "les Indiens ont tué Paul dans leur maison" }\end{array}$

Quand l'agent de 3ème personne agit sur soi-même, c'est la préposition ergative qui reçoit la marque $3 \mathrm{REFL}$ :

(14) bure

bure kune si-pa $\quad$ di-na-ho ?
mauvais INT $\quad$ 3-être tué
"est-ce que c'est un péché de se tuer?"

(15) pa-kri di-na-ho

être tué-PERF 3REFL-ERG-même

"il s'est tué"

\section{Nominalisation relative}

Comme je l'ai indiqué précédemment (1.), il y a dans cette langue une nominalisation relative pour les verbes des phrases intransitives et deux nominalisations relatives pour les verbes des phrases transitives. L'une de ces dernières réfère au patient et est morphologiquement identique à celle des phrases intransitives, laquelle réfère à l'expérient L'autre nominalisation réfère à l'agent et se distingue morphologiquement par l'ajout d'un préfixe $u$ Toutes les nominalisations sont formées avec le suffixe -ri NOMINALISATEUR et le préfixe $d$ - /di- mais celle de l'agent ajoute le préfixe $u$ - entre le thème verbal et le préfixe pour 3REFL : di-kangi-ri "celui qui est bon", di-te-ri "celui qui vient", di-ña-ri "celui qui meurt", di-pa-ri "celui qui est tué", mais $d-u-p a-r i$ "celui qui tue". Exemples :

(16) $u d z a$ di-di-ri no ware

couteau 3REFL-donner-NOM ERG curé

"le coulcuu que le curé a donné"

(17) ware $\quad d$-u-di-ri $u d z a$
curé 3 REFL-AG-donner-NOM
"le curé qui a donné le couteau" 
(18) pero di-pa-kri-ri $\quad h i-\tilde{n} a$

Pierre 3REFL-être tué-PERF-NOM 1-ERG

"Pierre, que j'ai tué"

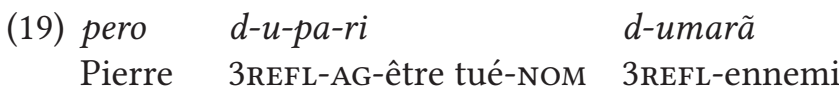

"Pierre qui a tué son ennemi"
(20) koho di-le-li
di-do-ho
i-dzene i-le
celui 3REFL-se.fâcher-NOM 3REFL-DAT-même 3-par.peur 3-se.fâcher
ñiño $i-d o o$
Dieu 3-DAT
"celui qui se fâche contre soi-même pour que Dieu ne se fâche pas contre lui" (exemple dzubukuâ)

\section{Conclusion}

Les faits présentés ci-dessus montrent que le Karirí présente une morphologie ergative et aussi une syntaxe ergative. Dans les phrases transitives, la préposition no (avec les allomorphes - na et - na en conjonction avec les marques de personne) est la marque du cas ergatif, tandis que le syntagme nominal non marqué est absolufif. Tous les verbes sont fléchis seulement pour la personne absolutive (expérient) et l'accord se fait exclusivement avec lui. La relativisation, en distinguant l'absolutif et l'ergatif, est une claire évidence de l'eigativité syntaxique du Karirí.
Abréviations
AG agent
COMPL complément
CONC concession
DUR duratif
FUT futur
INT interrogation
$\mathrm{N}_{\mathrm{AG}}$ nom/syntagme nominal agent
$\mathrm{N}_{\mathrm{EXP}}$ nom/syntagme nominal expérient
NEG négation
PERF perfectif 
PL pluriel

$\mathrm{V}_{\mathrm{IN}} \quad$ verbe intransitif

$\mathrm{V}_{\mathrm{TR}} \quad$ verbe transitif 